NASA Technical Memorandum 110359

\title{
The Development of the Final Approach Spacing Tool (FAST): A Cooperative Controller- Engineer Design Approach
}

Katharine K. Lee, Sterling Software, Inc., Palo Alto, California

Thomas J. Davis, Ames Research Center, Moffett Field, California

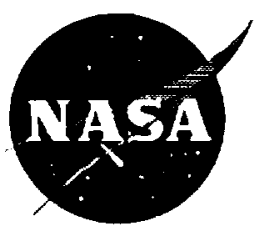

National Aeronautics and

Space Administration 


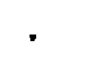

.

.

•

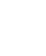




\title{
The Development of the Final Approach Spacing Tool (FAST): A Cooperative Controller-Engineer Design Approach
}

\author{
KATHARINE K. LEE* AND THOMAS J. DAVIS
}

Ames Research Center

\begin{abstract}
Summary
Historically, the development of advanced automation for air traffic control in the United States has excluded the input of the air traffic controller until the end of the development process. In contrast, the development of the Final Approach Spacing Tool (FAST), for the terminal area controller, has incorporated the end-user in early, iterative testing. This paper describes a cooperation between the controller and the developer to create a tool which incorporates the complexity of the air traffic controller's job. This approach to software development has enhanced the usability of FAST and has helped smooth the introduction of FAST into the operational environment.
\end{abstract}

\section{Introduction}

The development of an automation system for assisting terminal area air traffic controllers in efficiently managing and controlling arrival traffic has long been an objective of researchers and engineers. A fundamental issue for researchers in the development of such automation tools is to build functionalities and user interfaces that enhance the air traffic controllers' ability to perform well in their job. Early efforts in the automation of terminal air traffic control was presented by Martin and Willett (1968). Their system provided speed and heading advisories to controllers to help increase spacing efficiency on final approach. Although traffic tests of the system showed an increase in landing rate, controllers found that their workload was increased and rejected the system (Martin and Willett, 1968). An examination of the concept suggests that while some aspects of the design were sound, its acceptance was limited by the technology of the time period, especially the lack of an adequate controller interface. More recently, however, several automation systems have found their way into operational use in Europe due in large part to the introduction of modern computer processing and interfaces, and also because of more careful design approaches (Volckers, 1990; Garcia, 1990). In addition, recent real-time simulation studies have confirmed the potential for increasing landing rates

\footnotetext{
${ }^{*}$ Sterling Software, Inc., Palo Alto, California.
}

with the assistance of active advisories for controllers in the terminal area (Davis et al., 1991; Credeur et al., 1993).

A candidate system for the automated management and control of terminal area traffic, referred to as the Center TRACON Automation System (CTAS), is under development at NASA Ames Research Center in collaboration with the Federal Aviation Administration's (FAA's) Terminal Air Traffic Control Automation Program Office. The elements comprising the CTAS are the Traffic Management Advisor (TMA), the Descent Advisor (DA), and the Final Approach Spacing Tool (FAST) (Erzberger et al., 1993). The advisories generated by these tools assist controllers in handling aircraft arrivals starting at about $200 \mathrm{n} . \mathrm{mi}$. from the airport and continuing to the final approach fix. Recently, the elements of the CTAS system have been evaluated in a series of real-time simulations at NASA Ames Research Center and in field testing at facilities serving the Denver, Colorado, and Dallas/Fort Worth, Texas, areas.

This paper describes a cooperative effort between software developers, human factors specialists, and air traffic controllers to develop FAST. The main function of FAST is to provide landing sequence, landing runway assignments, and speed and heading advisories that help controllers manage and control arrival traffic and achieve an accurately spaced flow of that traffic onto the final approach course (Davis et al., 1991; Davis et al., 1994). This paper emphasizes the role of the air traffic controller in FAST development, from providing direction into the controlling strategies incorporated into the FAST algorithms. This paper also describes the use of the Controller Acceptance Rating Scale to track the controllers' perspective of the overall system through the development of FAST.

\section{Controller Input into FAST Development}

The CTAS development process has differed from more "traditional" approaches to software development by incorporating the expertise of the end-user at the beginning stages of development. Air traffic controllers have been involved in simulations at NASA Ames Research 
Center, the FAA Technical Center in Atlantic City, New Jersey, and in evaluation activities at the operational facilities into which the initial deployment have been targeted. The extensive involvement of controllers increases their understanding of the software and engineering constraints, and helps them to focus their expertise and provide input that is maximally useful to the software developers (Sanford et al., 1995). In addition, early end-user involvement also helps to integrate human factors issues during design, which can help improve system usability (Small, 1994). Larger questions of the suitability of a system, or how well the system provides for the users' problem-solving requirements (Harwood and Sanford, 1993), can also be addressed with early enduser involvement. The development, and ultimate demise, of the Advanced Automation System (AAS) provided numerous examples of design problems that could have been ameliorated with earlier controller input. Small (1994) describes how controllers involved toward the end of development were inappropriately focused on interface, rather than operational elements of the design. In addition, controller involvement late in the development process created an environment in which the controllers and the developers saw each other on opposite sides. This made reaching compromises much more difficult, as developers did not understand the operational needs of the user, and the controllers viewed development constraints they encountered as arbitrary (Small, 1994).

FAST simulations have been conducted at the Ames Automation Laboratory, where the software was originally developed and tested. Controller participation in these simulations, and other design activities, has helped to shape the requirements, test the software, and provide input into human factors issues as well as insight into controller strategies to reduce workload and increase throughput efficiency. In addition to a pool of local, retired controllers, controller personnel from the Dallas/ Fort Worth (DFW) TRACON, where the initial FAST testing and deployment will occur, have been evaluating FAST. DFW facility representatives, including Union members and managers, have been encouraged to participate in the software cvaluations. In total, three groups of controllers were incrementally involved in the FAST simulations.

The simulations consisted of traffic scenarios displayed on radar screens with FAST advisories added to the radar display interface. The traffic scenarios were created from recordings of live radar traffic from DFW. Two feeder controller positions (East and West) and three final controller positions (for the three arrival runways) were used for simulating DFW operations. Each arrival sector controller worked from a separate radar display position. The level of traffic during the 1 to 1.5 hour simulations was approximately 80 to 100 arrival aircraft per hour, reflecting the actual "rush" durations and traffic levels at DFW. Controllers were provided with headsets through which they issued commands to pseudo pilots, located in another part of the laboratory. The controllers were able to accomplish all of the basic entries and inputs into the system that would be expected in the real facility, such as taking handoffs and changing runway assignments.

Developers monitored the simulations in the laboratory; following simulations, debriefings were held to allow the controllers and developers to discuss key decisions made during the simulations, and explain any observed problems. All of the simulation outcomes were recorded for later review, and the debriefing sessions often included a replay of the simulation.

FAST has also been evaluated by controllers using a technique known as "shadowing." Shadowing involved viewing the live operational activities in real time, with FAST operating in the background, superimposing its advisories on an auxiliary computer display. Shadowing enabled controllers and developers to view the effects of the controllers' current procedures and how the traffic outcome was influenced by the presence of FAST advisories. Shadowing activities occurred in a testing environment near DFW, where radar communications were also monitored to provide a more complete picture of the traffic flow and decision-making activities.

\section{Phases of Controller Involvement}

Figure 1 shows the development of FAST through varying levels of development milestones and controller involvement. This paper concentrates on the three phases of controller involvement in simulation, from the initial software development to the preparation for the operational test.

Three different controller teams participated in the three levels of simulation fidelity. Each group of controllers provided increasingly more refined information in their evaluations. The first level, which required the initial assessment of new functionality and new displays, utilized the expertise of a group of local, retired controllers and pilots. The second level focused on expanded functionality and site-specific issues and incorporated the participation of a small cadre of controllers, or System Development Team (SDT), who represented the DFW facility. The third level increased the amount of feedhack and input into refining the FAST functionality and determined levels of acceptability for limited field testing, utilizing the expertise of a larger group of controllers from DFW. 


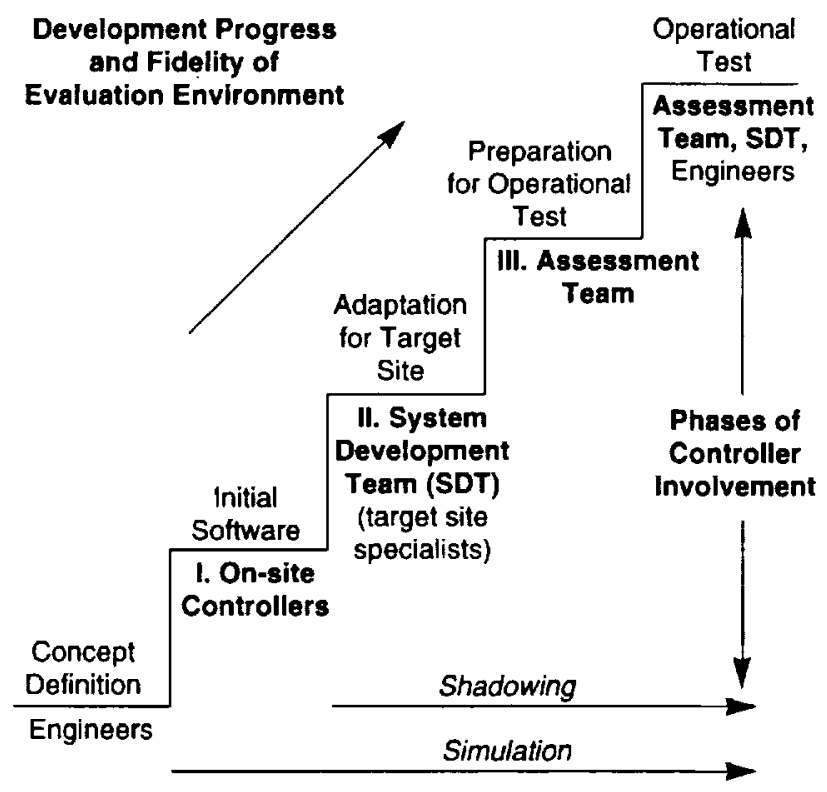

Figure 1. Controller involvement in the context of FAST development milestones.

\section{Phase I: On-Site Controllers}

The first level of FAST development involved creating a system prototype in which new functionality was first tested and graphical user interfaces were generated. Simulations were conducted several days a week with on-site controllers, most of whom were retired from regional facilities in the San Francisco Bay Area. Feedback from this early testing helped direct the iterative development of FAST.

The simulations with the on-site controllers enabled the developers to gauge the software's performance under basic, day-to-day testing conditions. The on-site controllers provided an indication of how air traffic controllers as a whole would react to the displays, controlling strategies, and advisories incorporated into the software. From a human factors standpoint, they provided the most input at the level of FAST's usability, determining if basic information could be extracted under simulated operations. They also provided some input on suitability issues, evaluating whether the information provided by FAST was appropriate for its intended use. In addition, the researchers benefited from on-site controller participation in that they were able to conduct studies under fairly well-defined conditions to determine the impact of FAST on controller workload. These studies showed that FAST reduced the level of self-reported workload by the air traffic controller (Lee et al., 1995) as well as reducing the number of commands issued to aircraft (Slattery et al., 1995).
It was clear, however, that the development of FAST functionality soon required more specific input from the DFW TRACON controllers, once basic concepts of air traffic control (ATC) were incorporated in the software. The on-site controllers' input into basic ATC concerns and attitudes was often accurate, but their understanding of the operations at the DFW TRACON was limited. As a result, the SDT was brought into the development process.

\section{Phase II: System Development Team}

The second phase of FAST development incorporated the expertise of a cadre of three controllers, one area supervisor, and one training specialist from the DFW TRACON. These controllers formed the SDT. The SDT provided the developers with guidance about the specific procedures of the operational environment and an understanding of operations under the high traffic levels that are common to DFW. The SDT participated in formal software evaluations at NASA Ames and the FAA Technical Center approximately every 6 to 8 weeks, depending upon milestones in the software design.

Split of FAST functionality- One of the SDT's pivotal inputs into FAST development was to split FAST functionality into two parts. FAST was originally conceived to provide a suite of advisory information for the TRACON sector controllers. This information would enable the TRACON controller to efficiently sequence and space arrival traffic by providing sequence and runway advisories. In addition, speeds and headings were provided to efficiently meet the sequences and runway assignments. Following an early evaluation, the SDT recommended that FAST be split into "passive" and "active" stages. Passive FAST was the portion of the software that provided only the sequence and runway advisory information and Active FAST was the portion where speed and heading advisories would be added. Together with the developers, the SDT decided that Passive FAST would be tested first.

In the early months of FAST development, the SDT was very cautious toward FAST and their recommendation to test Passive FAST first was directly attributable to this cautious attitude. It is possible that when the SDT first began to evaluate FAST, the utility of the FAST advisories was not fully developed, and hence the benefits were not as obvious as they have become. However, the SDT's acceptance of FAST increased significantly in the three years of their involvement in FAST development, and as a number of their concerns outside the software's capability (described below) were raised and addressed. This suggests that their initial apprehension toward 
developing full FAST capability was due to issues in addition to the immaturity of the software.

There were a number of reasons for the SDT's initial hesitancy. First, from the facility's perspective, the involvement of the SDT members could have implied that the facility's controllers were inefficient and that the abilities of the controllers as a whole were being called into question. It was easy to draw the conclusion that the development of ATC automation would prompt the FAA to reduce the controller workforce in response. Second, the SDT was clearly responsible for the outcome of FAST; thus the SDT's reputation as seen by the facility would be at stake. Third, the SDT had concerns about liability. The FAA could decide that FAST should dictate commands to the air traffic controller, yet the controller (and not the software) would be ultimately responsible for the consequences of the FAST advisories. Finally, the SDT was con cerned that FAST would automate the interesting aspects of the controller's job and thus reduce job satisfaction.

These concerns were not all obvious in the beginning of the development process. Some of the concerns the controllers discussed directly; other concerns were elicited after many months of development. The developers learned to provide reassurance to the controllers in addition to promoting the potential benefits of FAST. Also, the developers earned the trust and respect of the SDT through demonstrating a thorough understanding and appreciation of ATC in general, and DFW operations in particular. This knowledge helped the controllers and the developers work together to address the SDT's con cerns, resulting in changes to the software, or making compromises when software changes could not be accomplished.

SDT input into FAST algorithms- The SDT controllers hoped that FAST could provide a reduction in controller workload. However, it quickly became clear that while a basic element of workload was the number of aircraft for which a controller was respon sible, other variables, such as the types of aircraft involved, the characteristics of the sector's airspace, and the amount of coordination between positions, had to be considered. Consequently, the SDT showed that some FAST advisories, while intended to reduce controller workload by reducing delay, sometimes made the traffic situation more complicated from the controller's perspective.

The SDT also pointed out situations where FAST algorithms produced procedural obstacles and con trolling strategies that were considered unfamiliar or unfavorable. These issues required the developers and the controllers to work together to determine how the advisories were perceived and what could be done to minimize the impact on coordination and workload. In some cases, the SDT

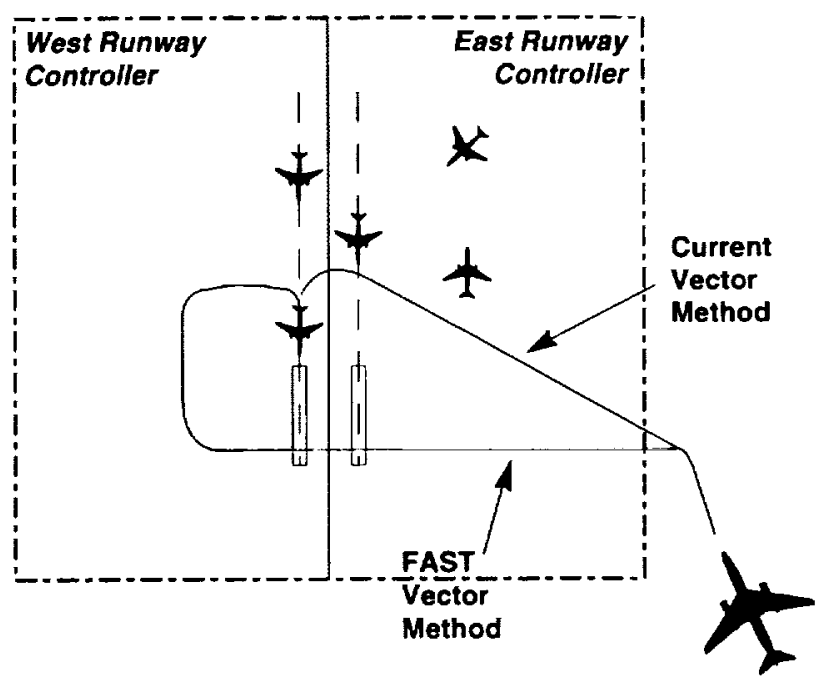

Figure 2. Possible routes for Southeast cornerpost arrival traffic.

discovered that the unfamiliar strategies FAST suggested were beneficial ones. For example, FAST would propose that aircraft from the Southeast cornerpost fix be vectored over the top of the airport to a West-side runway. The current procedures would have specified a different route to the West-side, or would have directed the aircraft to the East-side runway, as shown in figure 2. FAST's over-thetop vectoring was not typically favored by the facility, but the SDT found that this routing could provide benefits to their overall operations by decreasing the workload of the East runway controller.

Shadowing observations were a key turning point in the SDT's assessment of the software; they became an evaluation environment in which the benefits of FAST advisories became very evident. Figure 3 depicts a situation observed in many cases at DFW where the arrival rush side of the airport would be overloaded. More experienced controllers would have balanced the runway loading better than that $\mathrm{sec}$ in figure 3 , with vectoring strategies similar to those described earlier. By comparing the outcomes of FAST advisories to actual facility operations, the SDT and the developers could see where FAST could provide increased flow and efficiency as well as help determine where FAST needed to adjust for facility procedures.

After two years of SDT evaluations, it became clear that the SDT was providing input that was native to their own stylc of traffic control that was not neces sarily representative of DFW as a whole. As more advanced development issues were encountered, further input from the facility would be needed. In addition, FAST development was nearing a phase in which an operational test would be 


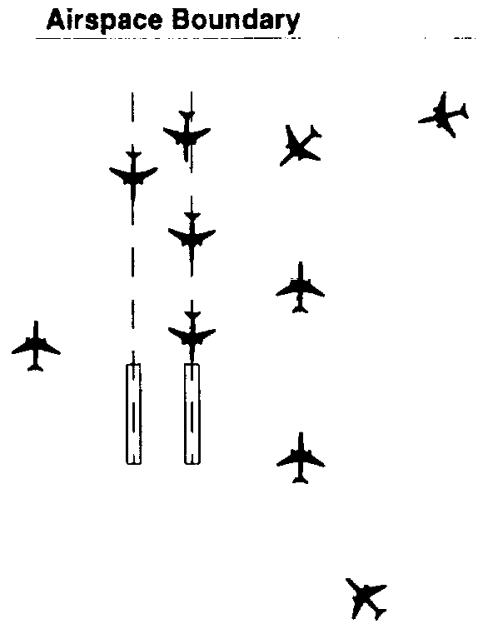

Figure 3. Shadowing observations demonstrating uneven runway balancing.

required. In order to conduct such a test, a larger group, or assessment team, of DFW controllers needed to be assembled and made proficient in using FAST.

\section{Phase III: Assessment Team}

The Assessment Team was composed of six controllers (three from each specialty) and two area supervisors from DFW TRACON. The Assessment Team participated in simulations at NASA Ames and the FAA Technical Center and will be participating in the upcoming Limited Operational Demonstration at DFW. The Assessment Team brought a wider range of skill, expectations, and viewpoints to the FAST development process. In their initial introduction to FAST, some of the Assessment Team members were pleasantly surprised and impressed that FAST could increase their throughput capacity without making them feel taxed. Others felt that they operated quite well without the need for added automation. As with the SDT, the developers had to work with the Assessment Team to come to an understanding of where the benefits of FAST's advisories and strategies could be realized. While the Assessment Team also introduced new controlling strategies not previously encountered, they also saw the benefits of changing their controlling strategies in the operational environment based on what they had seen in FAST simulations.

The Assessment Team's main goal was to determine when FAST would be ready for a limited operational test. But because developing ATC automation with active controller input was a new process within the FAA, there were no established guidelines to help determine system readiness and acceptability. Furthermore, the benchmarks of acceptability from the developer's perspective did not sufficiently address the controller's sense of workload. It was clear that qualitative measures of workload and acceptance had to be assessed along with quantitative measures of reduced delay and increased runway throughput.

\section{The Controller Acceptance Rating Scale (CARS)}

Based on the need for a measure of acceptability, the Controller Acceptance Rating Scale (CARS) was developed by the human factors specialists (fig. 4). The CARS was an adaptation of the Cooper-Harper Rating Scale for pilot assessment of handling qualities of aircraft (Cooper and Harper, 1969). CARS is still being assessed and validated at NASA Ames.

The original Cooper-Harper scale measured the performance of the pilot and the vehicle working together; this merging of the pilot and the vehicle defined the system being evaluated (Harper and Cooper, 1986). In adapting this scale to the ATC environment, this concept of a system including the user, the software, and the hardware was preserved. In addition, changes were made to the scale layout itself: compared to the original CooperHarper scale, the scale was reordered so that a rating of "l" was the worst performance level, and " 10 " was the highest performance level. This change was made to reflect that a lower number was to be associated with a lower, less acceptable rating, and the higher number was to be associated with a higher, more acceptable rating.

CARS was intended to provide the Assessment Team with a means for determining how well they and the FAST software performed together in controlling traffic in simulation. Following a simulation, each of the Assessment Team controllers viewed the scale, produced a rating, and gave an explanation for the rating. In addition, they were asked to provide a confidence indication of their rating, which could incorporate issues of the fidelity of the simulation, the amount of information they had available to make their rating, and other issues that might be external to the software advisories.

The four main rating levels described by CARS were controllability, tolerability, satisfaction, and acceptability. The controllers started at the top of the CARS diagram and first determined if the system was controllable. An uncontrollable system would imply safety violations such as near misses, collisions, or an inability to maintain separation. Such a system would require mandatory improvements.

If the system was rated as controllable, the controller then assessed the next level: tolerability. If the system was rated as intolerable, this implied major deficiencies and 


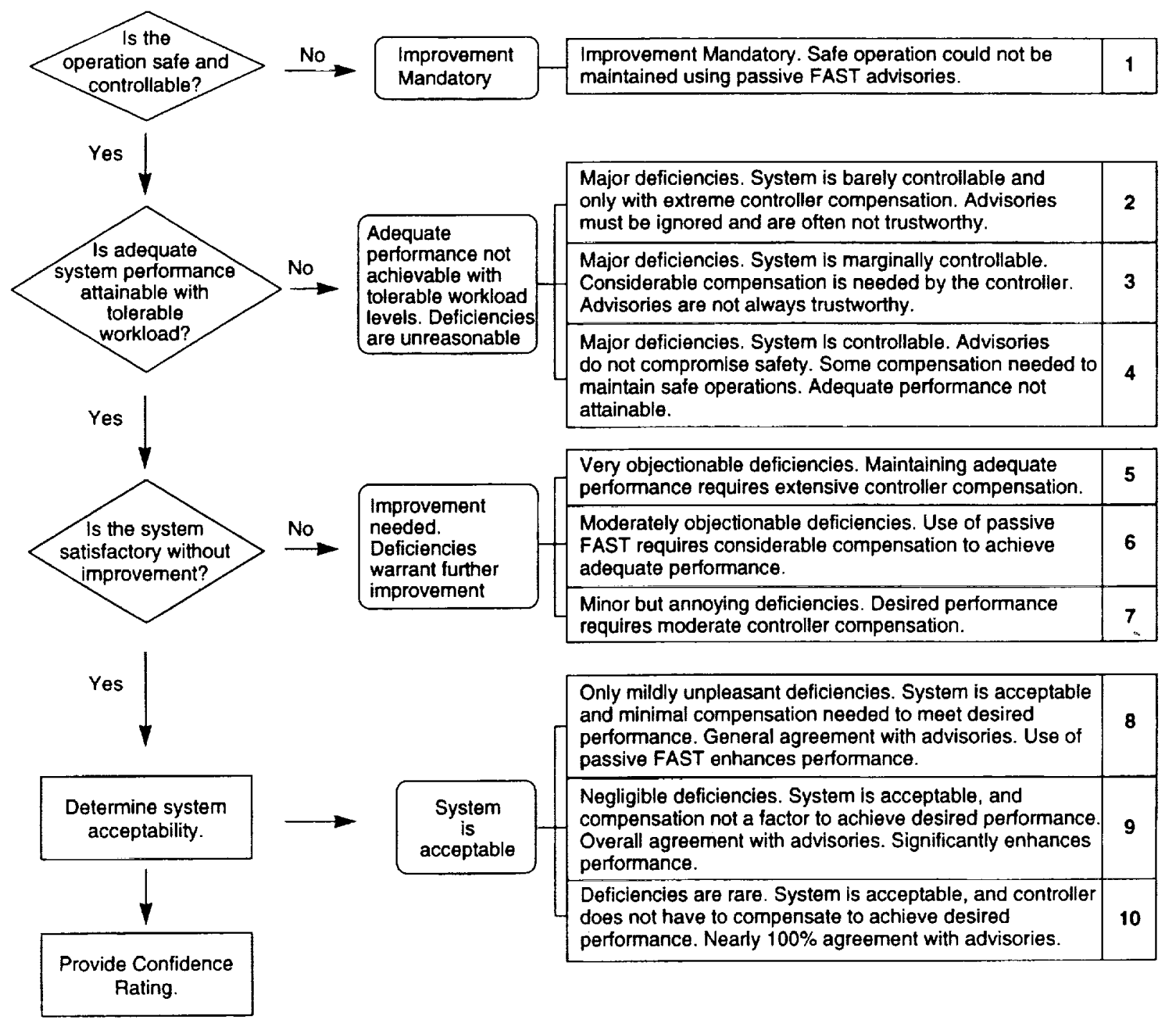

Figure 4. Controller Acceptance Rating Scale.

an inability to achieve adequate performance with tolerable workload levels. If the system was rated as tolerable, this implied a reasonable workload with manageable deficiencies, and the controller then assessed the next level: satisfaction. An unsatisfactory rating implied that the deficiencies warranted improve ment. If the system was considered satisfactory, however, the controller then rated the level of acceptability the system was able to achieve in the simulation. A confidence rating was obtained following the numerical rating. The Assessment Team was quite receptive to CARS.

To date, CARS has been used for one Assessment Team evaluation, in which the controllers participated in seven simulations with varied runway configurations typical to DFW operations. Each controller who participated in a simulation rated the system based on his own experience working traffic from his particular sector position.

Because CARS data have been collected from only one series of simulations, results are not presented here. In subsequent simulations and operational testing, the developers intend to continue to use CARS in order to track the progress of the software evaluation by the controllers. CARS has indications of being a useful measurement device, and its validation will be useful to other ATC software development processes. 


\section{The Benefits of a Cooperative Design Approach}

Closely linking the air traffic controller with the aeronautical engineer in developing ATC software helps to produce a system that will capture as much of the actual complexity of ATC as is possible prior to final field deployment. The process for incorporating air traffic controllers in the development of FAST allowed the FAST engineers to achieve a greater understanding and appreciation of the overall task of air traffic control, and helped them to create the most effective algorithms for generating efficient sequences and runway assignments. This process also allowed the controllers to become involved in understanding the software and engineering constraints under which the development occurred and helped provide focus in their feedback to the developers. Such a cooperative design approach was mutually beneficial to the controller and the engineer as both parties were able to satisfactorily understand each other's needs throughout development.

Early involvement of the air traffic controller was also extremely valuable for addressing human factors issues. The early and continuous involvement of controllers allowed interface and suitability issues to be tested and implemented throughout development. Issues such as information extraction, the assessment of workload, and, especially, the assessment of controller acceptance were able to be accommodated throughout development when changes could be made easily. Measurements taken by instruments such as the CARS have provided valuable insight into acceptance issues and also provided direction to the controllers on how to evaluate the system in the absence of outside guidelines.

By implementing the process described in this paper, the controllers have become more than just end-users awaiting a product. They are actively a part of the development of FAST and they clearly have demonstrated their enthusiasm for its success. Their active participation has contributed to the FAA's continued awareness and support of CTAS development. In addition, the controllers have become strong advocates of FAST in their facility, working to promote FAST as a beneficial addition to the controller's tools. Once the controllers themselves were satisfied that FAST was going to benefit the facility, and was not intended to replace the controller, they were able to provide reassurance to the facility that the concerns over job satisfaction and workload were being taken into consideration. Clearly, the input of controllers from the deployment site in early stages of development will be critical for upcoming operational testing. The controllers provided the developers with cues toward the overall facility culture, and have helped to prepare the facility for the introduction of new technology.

\section{Concluding Remarks}

The FAST development was a collaborative effort between air traffic controllers, developers, and human factors specialists. The development proceeded with increasing levels of expertise and complexity. The concept was first developed by engineers, then tested with retired controllers to gain early data on controller acceptability. Once this initial phase demonstrated benefits both to the controller and the air traffic control system, expert controllers from the initial deployment facility were introduced to gain specific expertise for the FAST system. These expert controllers drove the system development toward a usable system for their facility through real-time simulation and by shadowing live traffic operations with the FAST system. Finally, after a high degree of system fidelity was reached, a controller assessment team for the initial deployment site was brought into the final phases of development in order to prepare for an operational test. The assessment team controllers contributed to the final tuning of the system through real-time simulation and through the use of a new Controller Acceptance Rating Scale.

The complexity in developing automation aids for ATC is due to more than the complexity of modeling ATC strategies. The successful introduction of automation aids into the air traffic control environment must consider the impact on the air traffic controller in the form of changes to controlling strategies, workload, and job satisfaction. Early controller involvement helps to identify such concerns at a stage when changes to the software and education for the controller and developer can casily take place. In addition, early controller involvement helps to increase acceptance, which is the key measure of the software's success.

If the controllers' input is delayed until the end of the software development process, their sense of involvement is diminished and their operational concerns are likely to be ignored because of the inability to accommodate changes at later stages of development. In addition, preventing controllers from making inputs until the later stages of development assumes that the developers have anticipated all problems and that the facility doesn't need any time to grow accustomed to potentially large changes to the nature of its work.

By using a collaborative development approach, the FAST development team successfully integrated controllers into the development process. The controllers 
became integral to the development as much for their ATC expertise as for their input into directing the introduction of automation into the field. The support of the controllers helped focus the development of FAST, and the controllers themselves have been instrumental in the push toward field testing. This motivation and the direction provided by the controllers have helped the software become a tool that the controller will find useful, beneficial to the overall operations, and helpful for meeting the increasing demands of the ATC environment. The controllers' cooperative participation with the engineers has become key to the successful development of automation for the air traffic control system.

\section{References}

Cooper, G. E.; and Harper, R. P.: The Use of Pilot Rating in the Evaluation of Aircraft Handling Qualities. NASA TN D-5153, Washington, D.C., 1969.

Credeur, L.; Capron, W. R.; Lohr, G. W.; Crawford, D. J.; Tang, D. A.; and Rodgers, W. G.: Final-Approach Spacing Aids (FASA) Evaluation for Terminal-Area, Time-Based Air Traffic Control, NASA TP-3399. 1993.

Davis, T. J.; Erzberger, H.; Green, S. M.; and Nedell, W.: Design and Evaluation of an Air Traffic Control Final Approach Spacing Tool. J. Guidance Control and Dynamics, vol. 14, 1991, pp. 848-854.

Davis, T. J.; Krzeczowski; K. J.; and Bergh, C.: The Final Approach Spacing Tool. In: Proceedings of the 13th IFAC Symposium on Automatic Control in Aerospace-Aerospace Control '94, Palo Alto, Calif., 1994, pp. 70-76.

Erzberger, H.; Davis, T. J.; and Green, S. M.: Design of Center-TRACON Automation System. In: Proceedings of the AGARD Guidance and Control Panel 56th Symposium on Machine Intelligence in Air Traffic Management, Berlin, Germany, 1993, pp. 11-1-11-12.

Garcia, J.: MAESTRO-A Metering and Spacing Tool. In: Proceedings of the 1990 American Control Conference, San Diego, Calif., 1990, pp. 501-507.
Harper, R. P.; and Cooper, G. E.: Handling Qualities and Pilot Evaluation. J. Guidance, vol. 9, 1986, pp. 515-529.

Harwood, K.; and Sanford, B. D.: Evaluation in Context: ATC Automation in the Field. In: Human Factors Certification of Advanced Aviation Technologies, J. A. Wise, V. D. Hopkin, and P. Stager, eds., Embry-Riddle Aeronautical University Press, Daytona Beach, Fla., 1993, pp. 247-262.

Lee, K. K.; Pawlak, W. S.; Sanford, B. D.; and Slattery, R. A.: Improved Navigational Technology and Air Traffic Control: A Description of Controller Coordination and Workload. In: Proceedings of the Eighth International Symposium on Aviation Psychology, Columbus, Ohio, 1995.

Martin, D. A.; and Willet, F. M.: Development and Application of a Terminal Spacing System. Federal Aviation Administration, Rept. NA-68-25 (RD-68-16), Aug. 1968.

Sanford, B. D.; Harwood, K.; and Lee, K. K.: Tailoring Advanced Technologies for Air Traffic Control: The Importance of the Development Process. In: Proceedings of the Eighth International Symposium on Aviation Psychology, Columbus, Ohio, 1995.

Slattery, R. A.; Lee, K. K.; and Sanford, B. D.: Effects of ATC Automation on Precision Approaches to Closely Spaced Parallel Runways. In: Proceedings of the 1995 AIAA Guidance, Navigation, and Control Conference, Aug. 1995.

Small, D.: Lessons Learned: Human Factors in the AAS Procurement. MP 94W000088, MITRE, McLean, Va., 1994.

Volckers, U.: Arrival Planning and Sequencing with COMPAS-OP at the Frankfurt ATC-Center. In: Proceedings of the 1990 American Control Conference, San Diego, Calif., 1990, pp. 496-501. 
Public reporting burden for this collection of information is estimated to average 1 hour per response. including the time for reviewing instructions. searching existing data sources, gathering and maintaining the data needed, and completing and reviewing the collection of information. Send comments regarding this burden estimate ar any other aspect of this collection of inlormation, including suggestions for reducing this burden, to Washington Headquarters Services, Directorale for intormation Operations and Reports. 1215 Jelferson Davis Highway. Suite 1204, Arlington, VA 22202-4302, and to the Oflice of Management and Budget, Paperwork Peduction Project (0704-0188). Washington, DC 20503.
1. AGENCY USE ONLY (Leave blank)
2. REPORT DATE
3. REPORT TYPE AND DATES COVERED
August 1995
Technical Memorandum

4. TITLE AND SUBTITLE

The Development of the Final Approach Spacing Tool (FAST):

A Cooperative Controller-Engineer Design Approach

6. AUTHOR(S)

$505-64-13$

Katharine K. Lee* and Thomas J. Davis

7. PERforming organization NAME(S) AND AdDRESS(ES)

Ames Research Center

Moffett Field, CA 94035-1000

8. PERFORMING ORGANIZATION

REPORT NUMEER

A-950081

9. SPONSORING/MONITORING AGENCY NAME(S) AND ADDRESS(ES)

10. SPONSORING/MONITORING AGENCY REPORT NUMBER

National Aeronautics and Space Administration

Washington, DC 20546-0001

NASA TM-110359

11. SUPPLEMENTARY NOTES

Point of Contact: Katharine K. Lee, Ames Research Center, MS 210-2, Moffett Field, CA 94035-1000

(415) 604-5051

* Sterling Software, Inc., Palo Alto, California.

12a. DISTAIBUTION/AVAILABILITY STATEMENT

12b. DISTRIBUTION CODE

Unclassified - Unlimited

Subject Category 04

13. ABSTRACT (Maximum 200 words)

Historically, the development of advanced automation for air traffic control in the United States has excluded the input of the air traffic controller until the end of the development process. In contrast, the development of the Final Approach Spacing Tool (FAST), for the terminal area controller, has incorporated the end-user in early, iterative testing. This paper describes a cooperation between the controller and the developer to create a tool which incorporates the complexity of the air traffic controller's job. This approach to software development has enhanced the usability of FAST and has helped smooth the introduction of FAST into the operational environment.

14. SUBJECT TERMS

Air traffic control, Automation, Human factors 15. NUMBER OF PAGES 


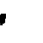

